\title{
High-Energy Proton-Proton Scattering in the Eikonal Model.
}

\author{
A. Capella, J. Kaplan. A. Krzywicki and D. Schiff \\ Laboratoire de Physique Théorique et Hautes Energies - Orsay \\ (Nuovo Cimento, $63 \mathrm{~A}, 141$ (1969))
}

i) In eq. (16) replace $\sqrt{\frac{-t}{s}}$ by $\sqrt{\frac{-t}{4 s}}$.

ii) Obvious corrections of the numbers on the ordinates of Fig. 5-8 should be made: these numbers should be $0.1,0.2$ ete. in Fig. 5 and $\pm 0.05,0.1$ etc. in Fig. 6-8.

iii) An error has been made in drawing the curves in Fig. 5. The actual value of the calculated polarization is twice smaller.

One comment is to be made in connection with iii). There is a systematic discrepancy between the polarization data of ref. $\left({ }^{20}\right)$ and those of ref. $\left({ }^{21}\right)$ (at 5-6 GeV/c). The latter results are confirmed by the recent data of the CERN-Orsay-Pisa collaboration. It is worth-while to notice that our model favours the CERN-Orsay-Pisa results. 